Instituto Internacional de Investigación y Desarrollo Tecnológico Educativo INDTEC, C.A.

DOI: https://doi.org/10.29394/Scientific.issn.2542-2987.2019.4.13.10.200-220

OAI-PMH: http://www.indteca.com/ojs/index.php/Revista Scientific/oai

Artículo Original / Original Article

\title{
Competencias Investigativas del Docente Construidas durante la Formación Universitaria
}

Autoras: Yizza María Delgado Nery De Vita Universidad Nacional Experimental "Rafael María Baralt", UNERMB yizzadelgadodevita@gmail.com Trujillo, Venezuela https://orcid.org/0000-0001-9193-7453

Rosilio Ramón Alfonzo Mendoza Universidad Nacional Experimental "Rafael María Baralt", UNERMB rosilioalfonzo@gmail.com Trujillo, Venezuela https://orcid.org/0000-0002-0430-850X

\section{Resumen}

La universidad en este momento, se constituye como el contexto propicio para encuentros e intercambio de saberes, mediados por lógicas y cosmovisiones salientes, que buscan su creencia y significación social. Este artículo científico es sustentado en el paradigma positivista, con enfoque cuantitativo. Tiene como objetivo general, crear principios metodológicos referentes a las competencias investigativas del docente, construidas durante su formación universitaria. Apoyada con referentes teóricos como los de Gallardo (2003); Ordóñez (2017), entre otros. La metodología es de tipo descriptiva, apoyada en un diseño de campo, donde se aplicará un estudio para docentes adscritos a la Universidad de Los Andes, Núcleo Universitario "Rafael Rangel" (ULA-NURR), estado Trujillo. De acuerdo con los planteamientos investigados en el artículo, se concluye que es de esperar que los estudiantes al momento de producir conocimiento en el proceso investigativo sean capaces de establecer relaciones de conexión entre teoría y práctica. Además, en el desempeño de su accionar investigador el estudiante deberá adquirir nuevos conocimientos para un eficiente desarrollo de las competencias investigativas y así puedan generar producciones científicas en pro de la sociedad del entorno al que pertenezcan.

Palabras clave: investigación; competencias del docente; formación profesional superior.

Fecha de Recepción: 15-10-2018
Fecha de Aceptación: 20-03-2019
Fecha de Publicación: 05-08-2019 


\title{
Teaching Researcher Competences Built during University Training
}

\begin{abstract}
The university at this time, is the ideal context for encounters and exchange of knowledge, mediated by outgoing logics and worldviews, seeking their belief and social significance. This scientific article is based on the positivist paradigm, with a quantitative approach. Its general objective is to create methodological principles concerning the investigative competences of the teacher, built during their university education. Supported by theoretical references such as those of Gallardo (2003); Ordóñez (2017), among others. The methodology is descriptive, supported by a field design, where a study will be applied for teachers assigned to the University of Los Andes, University Nucleus "Rafael Rangel" (ULA-NURR), Trujillo State. In accordance with the approaches investigated in the article, it is concluded that it is expected that students at the time of producing knowledge in the research process are able to establish relationships of connection between theory and practice. In addition, in the performance of its investigative actions the student must acquire new knowledge for an efficient development of the investigative competences and thus be able to generate scientific productions in favor of the society of the environment to which they belong.
\end{abstract}

Keywords: research; teacher qualifications; professional training.

Date Received: 15-10-2018
Date Acceptance:

20-03-2019
Date Publication:

05-08-2019 


\section{Introducción}

La temática a abordar en el presente artículo científico, se refiere a las competencias que debe abordar el docente, en el campo de la investigación, recibidas en su formación profesional superior. Además, se corresponde lo investigado con una investigación dogmática en construcción, sustentada en el paradigma positivista, con enfoque cuantitativo. Por esto, la actividad es considerada de alta derivación social, en los espacios universitarios y la sociedad.

En la posmodernidad, el sujeto inhabilitó y conjeturó los organismos y se impuso la emancipación, hubo un cambio notable en la personalidad del individuo, especialmente, en la juventud de la época, observándose una característica que les ofrecía herramientas preliminares, acordes al movimiento, además de almacenar búsquedas e indagaciones. No obstante, fue la investigación, un mundo que se reconcilió en el principal auge del joven posmoderno, transmitiendo eficientemente, una mayor cantidad de conocimientos supuestos y progresivos, adecuados al avance cognoscitivo, porque representan plataformas alusivas a las competencias del futuro.

Aunado a esto, la Organización de las Naciones Unidas para la Educación, la Ciencia y la Cultura (UNESCO, 1998), en la Conferencia Mundial sobre la Educación Superior, establece:

Promover, generar y difundir conocimientos por medio de la investigación y, como parte de los servicios que ha de prestar a la comunidad, proporcionar las competencias técnicas adecuadas para contribuir al desarrollo cultural, social y económico de las sociedades, fomentando y desarrollando la investigación científica y tecnológica a la par que la investigación en el campo de las ciencias sociales, las humanidades y las artes creativas (art. 1).

Esto supone, la importancia que conceden los organismos internacionales al tema de las competencias en investigación y en el presente 
artículo científico, son tomados en cuenta debido a que son relevantes porque se deben tomar en cuenta cómo intervienen en la construcción del aprendizaje que se adquiere en los contextos de la educación superior.

Asimismo, es significativo hacer referencia a lo expuesto en el Informe de la Ciencia perteneciente a la Organización de las Naciones Unidas para la Educación, la Ciencia y la Cultura (UNESCO), según su director Bokova (2015): “...pone de manifiesto que la investigación es un factor de aceleración del desarrollo económico y, a la vez, un elemento determinante en la construcción de sociedades más sostenibles y susceptibles..." (pág. 2).

Dicho de otra manera, se imagina al investigador como aquella persona denominada sujeto-investigador que, apoyado en su intencionalidad, curiosidad y criticidad comienza a buscar conocer, para compensar necesidades particulares, mutuas, materiales, científicas, en cuyo proceso va adquiriendo, acumulando nociones y prácticas.

En tal sentido, buscar conocimientos nuevos implica docencia y extensión, porque generar sapiencias derivadas de la investigación comprende acciones curriculares y planes de estudio, para enseñar a los estudiantes en su transitar formativo de los diferentes niveles educativos, en este caso el superior para luego ser transmitidos a la sociedad.

Aunado a esto, el objetivo a conseguir supone estudiar todo accionar docente enfocado, en transferir toda intuición generada en las distintas modalidades y niveles del sistema educativo superior venezolano. Es decir, pensar en el acto investigativo, no sólo desde la propia investigación sino idearlo desde el accionar pedagógico del profesor universitario para asumir una reflexión constante de éste en su labor de la cotidianidad.

Del mismo modo, el papel docente-investigador implica reflexionar permanentemente, asumir una actitud crítica sobre lo aprendido en su formación y notificar saberes con sus pares y estudiantes, para establecer sinergias entre lo que se aprende e investiga, además sobre lo que se enseña 
y el para qué. Imparcialmente, la figura del docente-investigador lleva a considerar al sujeto como un representante reflexivo capaz de articular la praxis docente con la investigación. Así, este estudio busca orientar, la formación basada en competencias, entendiendo los aspectos esenciales que se dan en tres aspectos fundamentales a juicio de Pérez (2012a):

El cognitivo que traslada a la aprehensión, adjudicación, comprensión e internalización del conocimiento; la puesta en práctica de las habilidades o destrezas que llevan a la aplicación del saber y el ser como aspecto más importante del proceso formativo dado el carácter actitudinal y comportamental (pág. 10).

Interpretando los supuestos, formar al estudiante por competencias lo prepara ontológicamente para su desenvolvimiento como ser social, con la alternativa de resolver problemáticas que son competencia de su entorno, es decir, fusionar lo laboral con lo académico. Esta nueva modalidad, ofrece al estudiante circunstancias para la inserción en campos laborales, que ameriten diversas preparaciones, acordes a necesidades contextuales.

\section{EI Problema}

La investigación se ocupa, del rol que el docente asume en determinado momento; es decir, no está mediado sólo por las condiciones espaciotemporales y funcionales que le corresponde desempeñar, están inmersas además las consideraciones ontológicas y epistemológicas que instituyen la matriz epistémica que apoya el interés de esta indagación. Ante tales planteamientos, en este momento el ejercicio docente se ve afectado, pues debe apoyarse en la visión del saber, donde sea capaz de observarlos para poder construir experiencias significativas como hoy se sugiere sean en colectivo, porque encontrará en su devenir conflictos, apatías históricas, además de predisposiciones contrarias.

El docente en la posmodernidad, debe estar plenamente identificado 
con las organizaciones universitarias y el entorno familiar, en lo que se refiere al logro del desarrollo integral del estudiante. Ser docente significa, tener la ocasión de enfrentarse día a día a una caja de admiraciones como: una sonrisa, lamento, acierto, una incógnita difícil de responder, situaciones que hacen de la profesión universitaria ser poseedor de momentos gratificantes y desafíos permanentes.

Ahora bien, el rol de investigador en los espacios universitarios ha sido analizado por diferentes organismos multilaterales a escala mundial y local, sugiriendo una nueva mirada en la acción instructiva aunada, a la acción investigativa, enfocadas como la razón de ser de la universidad como función primigenia. Es decir, la misión y oficio de estos entes, es originar conocimientos, lo que implica el desarrollo referente a: la dirección, alineación, anunciación y transferencia de esos saberes hacia la sociedad.

Esto lleva a plantear, el debate en el hoy y el mañana, visualizando que el ser, hacer y convivir del acto investigativo, infiere nuevos abordajes y mediaciones, adoptando posturas epistémicas no habituales que trasladan los cánones delimitados por el cientificismo, inclinándose hacia la alineación de sujetos crítico-transformadores y emprendedores, abocados a una praxis reflexiva, desde su interior como individuos sociales sistémicos. Al respecto, se puede observar en las universidades nacionales como la Universidad de Los Andes (ULA), la escasa conformación de grupos y líneas de investigación referente, a lo teórico-formal, lo que conlleva a la no formación de investigadores, principiantes a consolidados.

Debido a que éstas son causas que infieren para la no adecuación de espacios, para la socialización, confirmación de saberes, experiencias, emanadas de realidades específicas y necesidades sentidas. Igualmente, se observa que hasta el momento no existe una metodología única, para evaluar la productividad investigativa, científica y tecnológica, según Damiani (2010): "es oportuno abordar esta problemática desde otros enfoques para obtener 
representaciones más integrales y humanistas" (pág. 68).

De igual manera, provocar sapiencias en la sociedad actual, es polisémico, complejo porque manifiesta a distintas variables, elementos y situaciones, en su proceso. Además, esto traería como causas para ser insertado contextualizarlo, crear valores para evidenciar una cultura investigativa, como propósito para su legitimidad en los niveles educacionales, así como elementos para sustentar los perfiles del egresado de tipo psicosocial y emocional.

De cualquier manera, entre las consecuencias que puede transferir la problemática descrita, se deben superar vacíos conceptuales pertinentes a cómo se registran las experiencias donde se pueda enfocar en una visión crítica-reflexiva producto del esfuerzo investigativo del presente, articulándolo con aspectos que serán interesantes y centrales en el futuro; desconocen los vínculos que se establecen entre los saberes populares y los científicos.

En síntesis, desde estas afirmaciones se observa que hay desarticulación entre el acto de investigar y docencia en espera de generar elementos, que lleven a usar una metodología donde esto sea llevado a cabo como una vía, camino, para conseguir los objetivos planteados. Por lo anteriormente señalado, esta investigación pretende abordar la problemática vinculada con el posible incidente de las Competencias Investigativas del Docente Construidas durante la Formación Universitaria, en docentes de la Universidad de Los Andes, Núcleo Universitario “Rafael Rangel” (ULA-NURR).

Ubicados en dos secciones de educación, en todas sus menciones, para culminar con la generación de principios metodológicos para construir las competencias investigativas en su formación universitaria. A tales efectos, se plantearon unas interrogantes las cuales abarcan el objetivo general del estudio en su conjunto, a continuación, se plantea en forma breve el alcance de las mismas, determinando las adyacencias del evento a estudiar:

¿Cuáles son las competencias investigativas que aplican los docentes 
en su praxis educativa producto de su formación universitaria?; ¿Qué aspectos teóricos metodológicos de las competencias investigativas están presentes en el docente en su praxis educativa?; ¿Cuáles serían las relaciones entre las competencias investigativas del docente y su formación universitaria desde su praxis educativa?; ¿Qué elementos emergen para la construcción de principios metodológicos de las competencias investigativas del docente construidas durante su formación universitaria?

\subsection{Objetivos de la Investigación}

\subsubsection{Objetivo General}

Generar principios metodológicos, referentes a las competencias investigativas del docente construidas durante la formación universitaria. para docentes adscritos a la Universidad de Los Andes, Núcleo Universitario "Rafael Rangel" (ULA-NURR), estado Trujillo.

\subsubsection{Objetivos Específicos}

1.- Identificar las funciones de la formación universitaria y la vinculación con su praxis educativa.

2.- Precisar los aspectos teóricos-metodológicos de las competencias investigativas del docente, a partir de la praxis educativa, construida en su formación universitaria.

3.- Establecer relaciones entre las competencias investigativas y la formación universitaria del docente en su praxis educativa.

4.- Conformar principios metodológicos que originen las competencias investigativas construidas por el docente en su formación universitaria.

\subsection{Referentes Teóricos}

\subsubsection{La Educación Universitaria}

Hablar de universidad conduce al término cosmos, sinónimo de unidad 
convertido en uno. En este momento, las universidades enfrentan el reto de ser la institución académica orientada al compromiso que posee con las tres tareas fundamentales, necesarias en todo proceso social como son: la instrucción y formación de nuevas generaciones; la investigación, creación, mantenimiento, difusión de conocimientos; y servir de modelo social en cuanto a organización comunitaria, intelectual y democrática del conocimiento. Por esto, una institución de Educación Universitaria debe responder a las necesidades inmersas en los contextos a las que pertenecen en cuanto, a si sus carreras son las necesarias, de calidad y sus programas están actualizados.

Esta responsabilidad social nace de la propia Ley de Universidades (1970), al expresar:

Las universidades son instituciones al servicio de la nación y a ellas corresponde colaborar en la orientación general de la vida del país mediante su contribución doctrinaria en el esclarecimiento de los problemas nacionales, de tal manera que la responsabilidad social de las universidades tiene una traducción específica, dada la naturaleza de esas instituciones (art. 2).

De este modo, la responsabilidad social de la universidad, es vista como creadora de sapiencias, donde su primordial instrumento para transmisión de la experiencia cultural, científica, acumulada por la humanidad, donde deberá proyectar su actividad académica de formar un hombre del hoy-futuro, que según Gradolí (2015), en su resumen del informe a la UNESCO de la Comisión Internacional sobre la Educación para el siglo XXI presidida por Jacques Delors, el cual afirma:

Fundamentalmente, la UNESCO contribuirá a la paz y al entendimiento mutuo entre los seres humanos al valorizar la educación como espíritu de concordia, signo de una voluntad de cohabitar, como militantes de nuestra aldea planetaria, que debemos concebir y organizar en beneficio de las generaciones futuras. En ese sentido, la Organización contribuirá a una 
cultura de paz (pág. 5).

En vista de esto, como seres humanos, hay un compromiso ineludible como profesionales y universitarios. Además, hay enormes e inevitables responsabilidades, no solo de buscar el conocimiento, de andar en la permanente búsqueda de él, de ejercer nuestra profesión de forma honesta, sino se tiene el deber de, crear las nuevas realidades que ofrezcan los ambientes donde fertilicen copiosos sueños.

\subsubsection{Formación Basada en Competencias para Investigadores}

Formar docentes por competencias avocados al trabajo investigativo, es proponerse metas para el logro de un profesional flexible, creador, con suficiente capacidad en resolución de problemas que afectan al entorno al cual se circunscriben aunado al compromiso de ser investigador. Visto de esta forma este trabajo busca orientar, al profesional sobre el ejercicio de las competencias investigativas, según Gallardo (2003):

Como una configuración construida y desarrollada por los investigadores en su contexto y ejercicio profesional. Incluye la solución de problemas del entorno donde muestra habilidades en el arte de investigar, a través de la cual logra incorporar lo nuevo que permitirá transformar la realidad, utilizando la tecnología; además comunica y dirige el proceso hacia la obtención de resultados de significativo impacto económico, científico, social y/o medio ambiental (pág. 11).

Es decir, para lograr la competencia investigativa se deben implementar estrategias para fortalecerlas. Asimismo, integrar saberes, haceres y el ser, aunado a vivencias, sustituir metodologías neutrales y adaptar contextos de aprendizaje acordes para la investigación. Para esto, el profesional que se precie de tener competencias investigativas debe desarrollar lo cognitivoafectivo y lo axiológico-actitudinal íntimamente ligado al acto creador.

Como puede observarse, la relación de lo cognitivo con la afectividad 
significa poder percibir la personalidad del sujeto, de forma indisoluble sintetizando dualidades en los procesos. Se entiende entonces, que la personalidad es indivisible, se fusionan los elementos cognitivos y afectivos, pero solamente desde el punto de vista investigativo. Por tanto, una caracterización de las competencias investigativas es que estos procesos se dan unidos, identificándose con alguna dimensión por lo que es válida su diferenciación y estudiarlo de forma aislada.

En cuanto, al desarrollo axiológico-actitudinal la educación universitaria debe estar comprometida en la formación de individuos integrales, cimentada en valores actitudes y conductas que inciten el ímpeto por la investigación, entendida según Pérez (2012b): "como un proceso de búsqueda, generación y construcción de conocimiento, sistemático, controlado, reflexivo y crítico, basado en el deseo de indagación, comprobación, comprensión y cambio de la realidad, la cual permite explicaciones lógicas, coherentes, innovadoras y creativas" (pág. 22).

A tal efecto, Rojas (2005), define la investigación: "como un proceso de construcción de conocimiento, de indagación, de interrogación requiere, así mismo, la construcción de sujetos que indaguen y se interroguen, que se expongan y se arriesguen en esa irracionalidad insegura que es la ciencia" (pág. 86). Dicho de otra manera, la cultura investigativa es ineludible para lograr conocimiento respecto de todos los saberes omitidos con respecto, a los procesos de investigación como el problema, formulación de hipótesis, dirigidos a elaborar conclusiones precisas para posteriormente, solucionar.

De manera concluyente, el docente que egresa de su formación universitaria debe tener una actitud positiva hacia la investigación, para que pueda manifestar el espíritu científico, para ello deberá generar preguntas, controversias, que le apasione la investigación, generando saberes creativos y traslade proyectos de investigación, aplicando métodos apropiados según la esencia y teoría acogidos. 


\subsubsection{Caracterización de las Competencias Investigativas}

Haciendo un poco de historia, el término de competencia fue introducido por Chomsky (2000): "para explicar las competencias lingüísticas, lo que ha permitido la construcción de una pedagogía eficaz para el desarrollo del aprendizaje de la lengua en el ámbito educativo" (pág. 29). De otra manera, lo importante radica en que para desarrollar competencias se condiciona por las vivencias, conocimientos y creencias desarrolladas al existir, que llevaron a definir personalidades influenciando directamente en actitudes y formas de comportarse.

Por otra parte, esto hace referencia al conjunto de conocimientos, habilidades, aptitudes y actitudes usados durante el ejercicio de una función académica haciendo describir a la investigación en la profesión docente. Asimismo, esta investigación asume como objetivo orientar la formación de docentes en su accionar, que forman y enseñan a los educandos de la ULANURR, estado Trujillo, para desarrollar capacidades, inherentes a solucionar los diferentes problemas de investigación observados en los distintos ámbitos a los cuales pertenecen.

Por esta razón, el docente en su misión de enseñar y formar al estudiante deberá tener ciertas competencias en cuanto a ser investigador, habrá de poseer conocimientos, habilidades, destrezas para ser capaz de realizar interrogantes, hacer registros, realizar procesos cognitivos como analizar, sintetizar, reflexionar en la manera cómo está desempeñándose en su praxis, escribir, producir y comunicar.

Ello implica, la importancia de adquirir las competencias investigativas, que fueron construidas en el transcurrir del proceso de formación universitaria para reforzarse en su práctica docente. Estas cualidades de competencias expuestas, se irán edificando a lo largo de la formación universitaria del docente de la ULA-NURR, como el caso presentado, y corresponderá a los sujetos objeto de estudio desempeñarlas. 
Asimismo, según Ordóñez (2017): "desde la perspectiva del investigador, las competencias desde el enfoque funcional en dimensiones complejas involucran el proceso de destrezas en encuentros de saberes con prácticas epistemológicas...en entorno en función para la vida de acuerdo a criterios para la investigación" (pág. 286).

\section{Marco Metodológico}

Los aspectos metodológicos sitúan el camino a seguir en la indagación del estudio presentado, por cuanto esos procedimientos son los que orientan cualquier indagación a realizar. Así, se presenta el enfoque elegido para la indagatoria, o modo de ver la realidad del sujeto investigado, acompañado del paradigma positivista con los respectivos planos o dimensiones de naturaleza del conocimiento: ontológico, epistemológico, metodológico y la relación intrínseca con el entorno al que pertenecen. Al igual el recorrido metodológico, cónsono con el método orientador de la investigación, detallando los procesos sistemáticos y empíricos.

\subsection{Orientación Epistemológica de la investigación}

El individuo se hace diferente, ante la necesidad de saber su entorno donde es participante. Esa insuficiencia, se manifiesta para desarrollar un conocimiento, producto de revelaciones durante siglos. De hecho, la indagación acreditada traza un camino formal generador de comprensión objetiva, representando la práctica genérica como teorización, imaginando diversos saberes estudiables desde diversas dimensiones.

Por esto, los esquemas del trabajo investigativo, obedecen rigurosamente a la alineación cognoscente del investigador, es decir, derivada del estilo de su manera de pensar, y de sus convicciones epistémicas, en base a las situaciones problémicas para resolver dificultades detectadas, llegando a convertirse en enfoque epistemológico. 
Según Palella y Martins (2010): "positivo es inseparable de relativo, de preciso, de cierto, de real. La filosofía positiva, se contenta con las realidades apreciables del organismo, a través de los sentidos, elimina toda inquisición sobre lo absoluto, por esto es relativo" (pág. 37); de manera tal, que la corriente paradigmática, se encuentra acogida en el enfoque cuantitativo.

Asimismo, se concibe el estudio mostrado bajo el enfoque cuantitativo, definido por Hernández, Fernández y Baptista (2006a): "el que utiliza la recolección, análisis de datos para contestar preguntas de investigación, probar hipótesis previas, confía en la medición numérica, el conteo y frecuentemente el uso de estadística para establecer con exactitud patrones de comportamiento en población" (pág. 112); es decir, este tipo de indagación permite medición y análisis de esquemas estadísticos relacionados con la variable competencias investigativas en la formación universitaria, de docentes egresados de la ULA-NURR.

\subsection{Tipo de Investigación}

En esta investigación se verifica la descripción de los datos con base en el contacto directo que se tiene. Por esto, el prototipo de investigación, para dar cumplimiento a los objetivos planteados. Sitúa el fin del estudio y la manera de recoger los datos necesarios. Por esto, Hernández, Fernández y Baptista (2006b), establecen que: "los estudios descriptivos buscan especificar las propiedades importantes de personas, grupos, comunidades o cualquier otro fenómeno que sea sometido a análisis" (pág. 112).

\subsection{Diseño de la Investigación.}

Al respecto, Balestrini (2006a): "es un plan global que integra de un modo coherente y adecuadamente correcto, técnicas de recogida de datos a utilizar, análisis previstos y objetivos" (pág. 131). Respecto al estudio presentado, la habilidad general para la recolección de datos a favor de los 
objetivos planteados, se orienta en un diseño de campo, porque los datos se acumularon claramente de los sujetos encuestados en el estudio.

Por esto, Arias (2006a): instituye que "la investigación o diseño de campo es aquella que consiste en la recolección de datos directamente de los sujetos investigados o de la realidad donde ocurren los hechos, es decir los datos primarios" (pág. 31); específicamente, los datos se recogerán mediante preguntas formuladas en un cuestionario dirigido a un grupo de docentes de la ULA-NURR.

\subsection{Población y Muestra Objeto de Estudio}

De acuerdo a la población, Hernández, Fernández y Baptista (2006c): "se trata de convertir en operativos o adaptables, los diversos elementos que intervienen en el problema a investigar" (pág. 175). Parafraseando a los autores, este quehacer compone un proceso apreciable que induce la verificación en concreto reflejada en el marco teórico, porque promueve la problemática en su realidad práctica, para conseguir la comprobación de los datos pertinentes.

Igualmente, Hernández, Fernández y Baptista (2006d): precisan la población como "un conjunto de todos los casos que concuerdan con una serie de especificaciones, diferencia que se presenta en investigaciones que no describen lo suficiente sobre sus características o consideran que la muestra se representa de manera automática" (pág. 95). Conscientes de este horizonte, el estudio aborda la cantidad de 6 docentes adscritos a la ULANURR. La muestra a juicio de Balestrini (2006b): indica que "es obtenida con el fin de investigar, a partir del conocimiento de sus características particulares, las propiedades de una población" (pág. 138).

\subsection{Técnica e Instrumento de Recolección de Datos}

Se utilizará como técnica la encuesta; en tal sentido, Hernández, 
Fernández y Baptista (2006e): establecen que "es aquella que permite determinar las opciones, actitudes, preferencias y percepciones respecto a los hechos o acontecimientos de interés" (pág. 299); en este caso, los autores antes citados la definen, como una técnica fundamental empírica en ciencias sociales.

En ese orden de ideas, respecto al instrumento se elaborará un cuestionario aplicando la Escala de Likert, con respuestas directas y cerradas con cuatro alternativas, a saber: siempre, algunas veces, casi nunca, nunca con las puntuaciones de 4, 3, 2, 1 para obtener los denominados datos primarios. A continuación, se muestra el Cuadro 1, con el Baremo respectivo.

Cuadro 1. Baremo para Analizar los Resultados del Instrumento.

\begin{tabular}{|c|l|l|}
\hline Puntuación & Alternativa de Respuesta & Criterio \\
\hline 1 & Nunca & Deficiente \\
2 & Casi Nunca & Regular \\
3 & Algunas Veces & Bueno \\
4 & Siempre & Muy Bueno \\
\hline
\end{tabular}

Fuente: Los Autores (2018).

\subsection{Validez}

Los instrumentos elaborados, se mostrarán a cinco expertos, que les corresponderá dar su validez, tres metodólogos y dos versados en la materia. Según Hernández, Fernández y Baptista (2006f): "La validez es el grado en que realmente un instrumento mide la variable que pretende medir" (pág. 280); es decir, la validación de contenido es permitida cuando posee criterio constructor, donde pueda establecer la correspondencia instrumento/teoría.

\subsection{Confiabilidad}

La confiabilidad determina la veracidad con la cual un cuestionario 
aplicado obtiene resultados semejantes en momentos de tiempo cortos. Para Arias (2006b): "la confiabilidad es el grado con el cual el instrumento prueba su consistencia, por los resultados que produce al aplicarlo repetidamente al objeto de estudio" (pág. 81).

\section{Conclusiones}

Los docentes egresados de la Universidad de Los Andes, Núcleo Universitario "Rafael Rangel" (ULA-NURR), en competencias investigativas constituyen una esperanza, si se conduce adecuadamente y con una posición clara de sus posibilidades. Es decir, cuando haya la producción del conocimiento de parte del estudiante del proceso de investigación sean capaces de establecer relaciones de conexión entre teoría y práctica. Un elemento crucial de este estudio hasta la presente, es que el docenteinvestigador pueda preocuparse del significado que representa la práctica investigativa.

Porque, además, se aconseja examinar la dialéctica- teórico-práctica en su hacer cotidiano, lo epistemológico, métodos, aunado al compromiso con la universidad, respecto, al rol que le incumbe desempeñar. Incentivar al estudiante para que logren generar producciones científicas a favor de la sociedad y del entorno al que correspondan, dirigidas a enriquecer los espacios que subyacen al entorno que les correspondan asumir como investigadores.

\section{Referencias}

Arias, F $(2006 a, b)$. Proyecto de Investigación: Introducción a la Metodología Científica. 5a. Edición, ISBN: 980-07-8529-9. Caracas, Venezuela: Editorial Episteme, 143 págs.

Balestrini, A. (2006a,b). Cómo se Elabora el Proyecto de Investigación. $7 m a$. Edición. Caracas, Venezuela: Consultores Asociados. 
Bokova, I. (2015). La investigación es clave para conseguir los Objetivos del Desarrollo Sostenible, según un informe de la UNESCO. Francia: Servicio de Prensa de la UNESCO. Recuperado de: https://es.unesco.org/news/investigacion-es-clave-conseguir-objetivosdel-desarrollo-sostenible-segun-informe-unesco

Chomsky, N. (2000). Los límites de la globalización. Barcelona, España: Ediciones Ariel.

Damiani, L. (2010). Conferencia Central. Seminario Internacional sobre la Producción de Conocimientos en la Academia: Posibilidades y Obstáculos. Caracas, Venezuela: Memorias de la Universidad Central de Venezuela - UCV.

Gallardo, O. (2003). Modelo de Formación por Competencia para Investigadores. Revista Contexto \& Educação, 18(70), 9-25, e-ISSN: 2179-1309. Recuperado de:

https://www.revistas.unijui.edu.br/index.php/contextoeducacao/article/v iew/1141

Gradolí, A. (2015). La educación encierra un tesoro. Informe a la UNESCO de la Comisión Internacional sobre la Educación para el siglo XXI presidida por Jacques Delors. Francia: Ediciones UNESCO. [Documento en línea]. Recuperado de:

http://neurofilosofia.com/wp-content/uploads/2012/12/Resumen-de-LAEDUCACION-ENCIERRA-UN-TESORO.pdf

Hernández, S., Fernández, C., \& Baptista, P. (2006a,b,c,d,e,f). Metodología de la investigación. México: McGraw-Hill.

Ordóñez, V. (2017). Competencias Investigativas Dirigidas a los Profesores de Ingeniería Eléctrica, desde un Modelo de Energía Alternativa. Revista Scientific, 2(5), 283-303, e-ISSN: 2542-2987. Recuperado de:

https://doi.org/10.29394/scientific.issn.2542-2987.2017.2.5.15.283-303 
Palella, S., \& Martins, F. (2010). Metodología de la investigación cuantitativa. Aragua, Venezuela: Fondo Editorial de la Universidad Pedagógica Experimental Libertador- FEDUPEL.

Pérez, M. (2012a,b). Fortalecimiento de las competencias investigativas en el contexto de la educación superior en Colombia. Revista de investigaciones UNAD, 11(1), 9-34, ISSN: 0124-793X. Recuperado de: http://hemeroteca.unad.edu.co/index.php/revista-de-investigacionesunad/article/view/770

Ley de Universidades (1970). Gaceta Oficial №1.429 Extraordinario de fecha 8 de septiembre. Caracas, Venezuela: Palacio Federal Legislativo. Recuperado de:

http://www.ucv.ve/fileadmin/user upload/comision electoral/Normativa legal/index.htm

Rojas, M. (2005). Investigar la Investigación. La práctica docente en la enseñanza de la metodología de la investigación en la universidad. Ibagué, Colombia: Universidad de Ibagué.

UNESCO (1998). Conferencia Mundial sobre la Educación Superior. París, Francia: Organización de la Naciones Unidas para la Educación, la Ciencia y la Cultura. Recuperado de: http://www.unesco.org/education/educprog/wche/declaration spa.htm 


\section{Yizza María Delgado Nery De Vita}

e-mail: yizzadelgadodevita@gmail.com

Nacida en estado Trujillo, Venezuela, el 25 de julio del

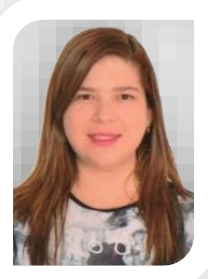
año 1984. Profesora ordinaria, escalafón-Asistente del área de Metodología de la Investigación del Departamento de Ciencias Sociales de la Universidad de Los Andes, Venezuela; Investigadora del Grupo en Estudios Críticos Culturales "Salvador Valero"; Miembro de la Comisión de Trabajos de grado de la carrera de Comunicación Social y miembro de la Comisión Curricular de la Carrera de Educación; Investigadora acreditada por el Observatorio Nacional de Ciencia y Tecnología; asesora y/o jurado evaluador de trabajos de grado en pregrado y postgrado. Por otra parte, tengo experiencia en producción radial, fotografía y uso de redes sociales para fines educativos. 


\section{Rosilio Ramón Alfonzo Mendoza}

e-mail: rosilioalfonzo@gmail.com

Nacido en Trujillo, Venezuela, el 27 de abril del año

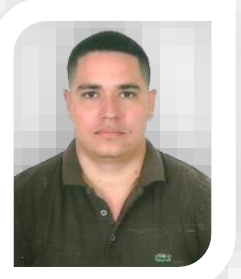
1977. Profesor ordinario, escalafón-asistente del área de prácticas profesionales del departamento de Ciencias Pedagógicas; Coordinador administrativo e investigador del grupo Geociencia y Taller "Abya-yala", línea de investigación Geohistoria; Asesor y/o jurado evaluador de trabajos de grado en pregrado y postgrado del Núcleo Universitario Rafael Rangel de la Universidad de Los Andes, Venezuela; Investigador activo del área de investigación histórica del Centro de Historia "Casa de los Tratados de Bolívar y Sucre", Trujillo, Venezuela; Coordinador del área de investigación histórica.

El contenido de este manuscrito se difunde bajo una Licencia de Creative Commons ReconocimientoNoComercial-Compartirlgual 4.0 Internacional 